\title{
Entscheidungsfindung für die klinische Praxis: Aktuelle Konzepte und Behandlungsalgorithmen mit Immun-Checkpoint-Inhibitoren beim Melanom
}

\author{
Dirk Schadendorf
}

Klinik für Dermatologie, Venerologie und Allergologie, Universitätsklinikum Essen, Essen, Deutschland

- Die S3-Leitlinie Melanom hat die Empfehlungen zur immunonkologischen (I-O) Therapie im Stadium IV an die neue Datenlage und Zulassungssituation angepasst und empfiehlt unter anderem bei Melanompatienten mit nicht resezierbaren Metastasen unabhängig vom BRAF-Status eine I-O-Therapie mit Checkpoint-Inhibitoren [1].

- In der für die I-O-Kombinationstherapie zulassungsrelevanten Studie CheckMate-067 waren Patienten mit einem Melanom im Stadium III oder IV eingeschlossen, die einen ECOG(Eastern Cooperative Oncology Group)-PerformanceStatus $0(73 \%)$ oder 1 (27\%) aufwiesen [2]. Wenn auch Daten zum Gesamtüberleben (OS) noch nicht auswertbar sind: Im Kombinationsarm (Nivolumab + Ipilimumab) erreichten nach 18 Monaten 46\% der Patienten ein progressionsfreies Überleben vs. $39 \%$ mit Nivolumab sowie $14 \%$ mit Ipilimumab in Monotherapie [3].

- Auch in CheckMate-069 wiesen die Patienten bei Studieneinschluss mehrheitlich einen ECOG-Performance-Status 0 oder 1 auf und nur 3 Patienten einen ECOG-Performance-Status $\geq 2$ [4]. Nach einem Follow-up von mindestens 2 Jahren zeigten Patienten mit BRAF-Wildtyp-Melanom eine 1-JahresOS-Rate von 79\% im Kombinationsarm (Nivolumab + Ipilimumab) versus $62 \%$ unter der Monotherapie mit Ipilimumab. Die 2-Jahres-OS-Rate betrug im Kombinationsarm 69\% versus 53\% im Ipilimumab-Arm (explorativer Endpunkt) [5]. Die guten Daten von Ipilimumab in der Monotherapie sind mit großer Wahrscheinlichkeit auch auf die nachfolgenden Therapien zurückzuführen, welche das OS entsprechend erhöhten.

\author{
Ist die I-O-Kombination eine Option? Potenzielle \\ Entscheidungskriterien für eine kombinierte \\ Immun-Checkpoint-Inhibitor-Therapie
}

\section{Allgemeine Voraussetzungen}

- Die I-O-Therapie mit Checkpoint-Inhibitoren sollte bei Melanompatienten im fortgeschrittenen (nicht resezierbaren oder metastasierten) Stadium geprüft werden.

- Die Therapieentscheidung ist von multiplen individuellen Patientenfaktoren abhängig wie Alter, allgemeiner Gesundheitszustand, Tumordynamik/Tumorlast, Mutationsstatus, LDH(Laktat-Dehydrogenase)-Ausgangswert und vorbestehenden Komorbiditäten bzw. Vorerkrankungen (z.B. pulmonal/kardial). Demgegenüber sind therapiebezogene Faktoren wie die Schnelligkeit des Ansprechens, Ansprechraten, Toxizitäten und Dauerhaftigkeit des Ansprechens abzuwägen.

- Die Patientenpräferenz sollte nach der Aufklärung insbesondere im Hinblick auf die Wirksamkeit und mögliche Nebenwirkungen im Sinne einer partizipativen Entscheidungsfindung berücksichtigt werden.

- Die I-O-Kombination ist angesichts der überlegenen Ansprech- und Überlebensraten gegenüber der I-O-Monotherapie bei Patienten ab der ersten Therapielinie in Betracht zu ziehen (vgl. Seite 4-5).

- Es gibt erste Hinweise dafür, dass ein frühzeitiger Einsatz der kombinierten I-O-Therapie bzw. I-O-Monotherapie günstiger für ein Langzeitansprechen des Tumors ist als der Einsatz

\section{KARGER}

(C) 2016 S. Karger GmbH, Freiburg 
nach Eintritt einer Resistenzentwicklung gegenüber einer BRAF-gerichteten Therapie [6,7].

\section{Kommentar}

Patienten mit hoher Tumorlast und/oder ECOG-Performance-Status $>2 / 3$ (z.B. Patienten mit drohender Akutsymptomatik, multiplen Knochenmetastasen, massiven Hirnfiliae, Schmerzen und oberer Einflussstauung) wurden nur begrenzt in den kontrollierten Studien eingeschlossen. Für die Mehrheit der Patienten impliziert die Entscheidung zur Kombinationstherapie allerdings eine um etwa 20\% erhöhte Chance auf ein dauerhaftes Therapieansprechen im metastasierten Stadium des Melanoms gegenüber der Monotherapie. Die kombinierte I-O-Therapie kommt dabei auch für Patienten mit erhöhten LDH-Ausgangswerten infrage (vgl. Seite 5).

Die Wahl der Therapie hängt zudem auch von der persönlichen Lebenssituation des Patienten und individuellen Präferenzen ab. Eine partizipative Entscheidungsfindung wird insbesondere für onkologische Situationen empfohlen, in denen die Behandlungsoptionen hinsichtlich des Behandlungsergebnisses vergleichbar sind, jedoch bei der Durchführung (z.B. Tablettenvs. Infusionsregime) oder bei den Nebenwirkungsrisiken deutliche Unterschiede bestehen. Fühlt sich der Patient allerdings überfordert oder wünscht er bei der Therapieentscheidung explizit keine Beteiligung, stellt die partizipative Entscheidungsfindung keine Option dar [8].

Eine vorbestehende Autoimmunerkrankung stellt eine relative Kontraindikation dar, die eine I-O-Therapie nicht ausschließen muss [9] und eine individuelle Risikoabwägung erforderlich macht.

In der bislang größten verfügbaren retrospektiven Auswertung einer Patientenserie bei fortgeschrittenem Melanom und komorbider, vorbestehender Autoimmunerkrankung sowie einer Behandlung mit Ipilimumab $(\mathrm{n}=30$, unter anderem Patienten mit rheumatoider Arthritis, Psoriasis, chronisch-entzündlicher Darmerkrankung) kam es bei der Hälfte der Patienten weder zur Auslösung eines neuen Schubs noch zu immunvermittelten Nebenwirkungen (immune-related adverse events (irAEs)) der I-OTherapie [9].

Die Frage, inwieweit Melanompatienten von höheren OSRaten profitieren könnten, wenn der Immun-Checkpoint-Inhibitor im Vorfeld einer BRAF-Inhibitor-Therapie bzw. möglichst frühzeitig eingesetzt wird, ist noch nicht hinreichend beantwortet: Während der vorangehende Einsatz von Ipilimumab mit einer OS-Verlängerung versus BRAF-Inhibitor $\Rightarrow$ Ipilimumab assoziiert war [6], fiel das Therapieansprechen auf Nivolumab im Rahmen einer offenen Phase-III-Studie auch bei Ipilimumabund BRAF-Inhibitor-vorbehandelten Patienten höher aus als auf eine Chemotherapie [10].

\section{Melanompatienten mit BRAF-Mutation}

Die I-O-Therapien mit Checkpoint-Inhibitoren in der Kombinations- oder Monotherapie stehen auch im fortgeschrittenen (nicht resezierbaren oder metastasierten) Stadium des Melanoms mit BRAF-Mutation (BRAFm) für die Behandlung in der ersten Therapielinie zur Verfügung. In der deutschen S3-Leitlinie zur Diagnostik, Therapie und Nachsorge des Melanoms (Stand: Juli 2016) werden die Immun-Checkpoint-Inhibitoren alleine oder in Kombination als gleichwertig mit den BRAF/MEK-Inhibitoren angesehen. Klare Entscheidungskriterien für oder gegen die eine oder andere Therapieoption liegen bisher nicht vor.

\section{Kommentar}

Derzeit kommt die I-O-Therapie als Erstlinientherapie bei ca. zwei Drittel der Patienten mit fortgeschrittenem BRAFm-Melanom infrage. Die I-O-Therapie mit Checkpoint-Inhibitoren in der Kombinations- oder Monotherapie stellt auch in dieser Patientengruppe eine Therapie der ersten Wahl dar, da das Therapieansprechen auf eine I-O-Therapie nach BRAF-Inhibitor-Therapie schlechter ausfallen kann als in Erstlinie [7, 11]. Zudem wurde kürzlich die Entwicklung von Kreuzresistenzen auf eine PD-1-Inhibitor-Therapie nach vorheriger MEK-Inhibitor-Therapie beschrieben [12].

Mit der Verfügbarkeit der Kombination von Nivolumab und Ipilimumab sowie den PD-1-Immun-Checkpoint-Inhibitoren hat sich auch das frühere Paradigma eines eher langsamen Therapieansprechens unter einer CTLA-4(cytotoxic T lymphocyteassociated antigen-4)-Immun-Checkpoint-Inhibition abgeschwächt. Die Kombinationstherapie kann somit auch Patienten angeboten werden, die auf eine schnelle Therapieantwort angewiesen sind.

\section{Potenzielle Behandlungsalgorithmen für Melanom- patienten mit Hirnmetastasen}

\section{Hintergrund}

Das maligne Melanom zeigt eine hohe Tendenz zur Hirnmetastasierung: Bis zu 20\% der Patienten jeglicher Tumorstadien entwickeln zerebrale Metastasen [13]. Im metastasierten Tumorstadium entwickeln 44\% der Patienten symptomatische Hirnmetastasen [14]. Ihre Prognose ist ungünstig: Die Überlebensdauer beträgt trotz Radiatio nur 3-13 Monate [15]. Zudem bilden Hirnmetastasen die häufigste Todesursache bei metastasierten Melanompatienten [16]. Die Studienlage zur Behandlung des hirnmetastasierten Melanompatienten ist in vielen Fragestellungen limitiert und die Evidenzlage zur Behandlung multipler Hirnmetastasen besonders dünn, denn bislang gehörten Hirnmetastasen in den meisten klinischen Studien zu den Ausschlusskriterien einer klinischen Studie. Dementsprechend sind auch kaum standardisierte bzw. evidenzbasierte Therapieoptionen und -empfehlungen in dieser Behandlungssituation verfügbar. Durch die erweiterten systemischen Therapieoptionen der letzten 5 Jahre und längeren Überlebensraten der Patienten gewinnt das 
klinische Management zerebraler Folgekrankheiten beim Melanom zunehmend an Bedeutung [14]. Zudem gibt es Hinweise, dass I-O- und zielgerichtete Wirksubstanzen auch bei der Behandlung von multiplen Hirnmetastasen eine Rolle spielen und sich als Bestandteil eines multimodalen Therapiekonzepts etablieren könnten [14].

\section{Mögliche Behandlungsalgorithmen bei Hirnmetastasen}

- Die Resektionstherapie bietet sich bei einzelnen (solitären) Hirnmetastasen an, die einer raschen Entlastung von symptomatischen raumfordernden Effekten bedürfen und chirurgisch zugänglich sind. Alternativ ist bei Patienten mit einzelnen oder wenigen Metastasen eine stereotaktische Einzeitbestrahlung (Radiochirurgie, Tumorgröße $<3 \mathrm{~cm}$ ) oder ein kombiniertes Vorgehen üblich. Liegt eine Einblutung vor, kommt die alleinige Resektion präferenziell infrage. Der Stellenwert einer adjuvanten Ganzhirnradiatio nach Lokaltherapie ist bislang nicht geklärt [16] und wird nicht empfohlen.

- Beim solitären Lokalrezidiv steht die Resektionstherapie oder Radiochirurgie zur Auswahl.

- Eine medikamentöse systemische Therapie bietet sich analog zu den Empfehlungen bei Metastasierung in andere viszerale Organe auch bei Patienten mit Hirnmetastasen an [17]. Beim BRAF-positiv getesteten Melanom (BRAFm) sollte primär die kombinierte BRAF-/MEK-gerichtete Inhibitor-Therapie angeboten werden. Beim BRAF-Wildtyp-Melanom stehen die Monotherapie mit einem PD-1-Inhibitor sowie die I-OKombinationstherapie (PD-1-Inhibitor plus Ipilimumab) zur Auswahl, wobei es bislang keine Vergleichsstudien zur Monoversus Kombinationstherapie bei Hirnmetastasen gibt.

- Beim BRAFm-Melanom kann als systemische Therapieoption die kombinierte BRAF-/MEK-Inhibition angeboten werden. Bei asymptomatischen Hirnmetastasen können auch I-OTherapieoptionen in Betracht gezogen werden. Ergänzend kommt eine Bestrahlung von Einzelläsionen infrage. Eine Ganzhirnradiatio stellt für Patienten mit multiplen Hirnmetastasen lediglich eine palliative Therapieoption dar.

- Retrospektiven Untersuchungen zufolge konnten Patienten mit Hirnfiliae eine deutliche Verbesserung des OS gegenüber der Radiatio allein erreichen, wenn Ipilimumab mit einer Radiatio kombiniert wurde $[14,18]$. Aktuell fehlen Kombinationsdaten von PD-1-Inhibitoren und Radiatio.

\section{Kommentar}

In der klinischen Praxis zeichnen sich beim zerebral metastasierten Patienten zwei zentrale Ausgangssituationen ab: entweder das Vorliegen solitärer oder multipler Hirnmetastasen. Bei der Therapieentscheidung stehen die Beschwerden (Symptome) des Patienten im Vordergrund. Zusätzlich ist bei der Therapieentscheidung zur Lokaltherapie die Größe der Metastase, das Vorliegen einer Einblutung sowie die Lage der Läsion zu berücksichtigen. Beim Rezidiv muss zudem auch die Vortherapie berücksichtigt werden. Lokale Rezidive werden bei mehr als 50\% der Patienten nach neurochirurgischer Resektionstherapie beobachtet. Der Stellenwert einer zusätzlich (bzw. adjuvant) durchgeführten Ganzhirnradiatio ist zunehmend umstritten, da weder die Effekte auf die lokale Tumorkontrolle ausreichend belegt sind noch das Risiko für neurokognitive Beeinträchtigungen ausgeschlossen ist [13].

Die medikamentöse systemische Therapie von Hirnmetastasen bietet sich im Rahmen eines multimodalen Managements in Ergänzung zu neuro- und radiochirurgischen Therapieoptionen an. Dabei können I-O-Substanzen ihre Therapieeffekte im Hirnparenchym auch dann entfalten, wenn sie die Blut-HirnSchranke nicht passieren, zumal aktivierte T-Zellen letztere überwinden [14, 19]. Von besonderem Interesse sind dabei «abskopale Effekte», d.h. durch kombinierte Bestrahlung und I-OTherapie induzierte Tumorregressionen, die sich nicht im Bereich der initial bestrahlten Region ereignen und auf ein synergistisches Potenzial von Radiatio und Immun-Checkpoint-Inhibition deuten $[14,18,20]$. Inwieweit sich unter der Kombination einer stereotaktischen Radiatio mit neueren systemischen Therapieoptionen das Risiko von Radionekrosen erhöht, bedarf weiterer prospektiver Analysen. Kasuistisch wurden Radionekrosen 6-18 Monate post Radiatio berichtet [21,22]. Klinisch sind Radionekrosen kaum von rekurrenten Hirnfiliae abgrenzbar [14, 23].

Vor dem Hintergrund der begrenzten Datenlage empfiehlt sich für Patienten mit multiplen Hirnfiliae grundsätzlich die Teilnahme an klinischen Studien. Derzeit sind mehrere Studien in Planung bzw. bereits angelaufen, die unter anderem die Wirksamkeit von PD-1-Inhibitoren und von kombinierten I-O- bzw. zielgerichteten Therapien sowie den kombinierten Effekt einer radiochirurgischen Intervention mit einer I-O- bzw. zielgerichteten Therapie evaluieren.

\section{Sicherheitsmanagement der I-O-Kombination: Worauf achten?}

\section{Interdisziplinäre Netzwerke etablieren}

- Mit der zunehmenden praktischen Bedeutung der I-O-basierten Therapieoptionen empfiehlt sich die Einrichtung lokaler Netzwerke an den behandelnden Zentren, die im Bedarfsfall die enge Abstimmung mit Ansprechpartnern aus den jeweiligen Fachdisziplinen ermöglichen. Je nach individuellem Adhärenzverhalten und Mobilität des Patienten kommt neben der ausführlichen Schulung des Patienten und seiner Angehörigen die wöchentliche Anbindung an das Zentrum (auch telefonisch) und/oder enge Abstimmung mit dem Hausarzt infrage (gegebenenfalls den Hausarzt dazu direkt kontaktieren).

- Um potenzielle irAEs (immunvermittelte unerwünschte Ereignisse) frühzeitig zu erkennen, empfiehlt sich eine engmaschige Überwachung und breite Ursachenabklärung, die im Einzelfall auch bei subklinischem Verdacht erfolgen sollte. 
Tab. 1. Einbindung der Fachkollegen in den Nachbardisziplinen und Aufklärung des Patienten und seiner Angehörigen

Etablierung eines interdisziplinären Netzwerks im Zentrum

- Gemeinsame Medikamentenstandards interdisziplinär absprechen und vor Beginn der Kombinationstherapie festlegen

- Aktives Nachfragen und intensiver Austausch

- Gegebenenfalls interdisziplinäre Fortbildungen zum Nebenwirkungsmanagement

Arzt vor Ort informieren und einbinden

- Behandler vor Ort aktiv ansprechen und gegebenenfalls über wichtige Laborparameter informieren

- Ansprechpartner für Hausarzt: Kontaktaufnahme bei kleinsten Anzeichen/ Verdacht auf irAEs (immunvermittelte unerwünschte Ereignisse) erleichtern (Abklärung und Nebenwirkungsmanagement erfolgt im Behandlungszentrum!)

- Patiententagebuch empfehlen

Patienten und Angehörige aufklären und einbinden

- Gemeinsame Aufklärungsgespräche mit Patient und Angehörigen über die Bedeutung einer sofortigen Kontaktaufnahme bei Symptomen

- Patientenkarte aushändigen/Unterstützung beim Patiententagebuch

- Individuelle Patientenadhärenz berücksichtigen, Kontrollintervalle festlegen und Kontaktmöglichkeiten anpassen (z.B. via E-Mail/Smartphone, Telefon)

Im klinischen Alltag sind beispielsweise Patiententagebücher hilfreich, um potenzielle irAEs (immunvermittelte unerwünschte Ereignisse) zu erfassen, ihre Schwere einzuschätzen und ihren Verlauf zu dokumentieren.

- Frühzeitig erkannt und behandelt, sind die meisten irAEs (immunvermittelte unerwünschte Ereignisse) im Allgemeinen gut beherrschbar und reversibel. Zum Management schwerwiegender irAEs (immunvermittelte unerwünschte Ereignisse) sind spezifische Behandlungsalgorithmen etabliert, die auch fester Bestandteil der Fachinformationen sind [24, 25].
- Im Rahmen der I-O-Kombinationstherapie empfiehlt sich insbesondere während der Kombinationsphase, in der Nivolumab plus Ipilimumab infundiert wird, eine engmaschige Überwachung, die sowohl die Klinik als auch das Labor einbezieht und idealerweise am spezialisierten Zentrum vorgenommen wird. Unterstützend können interdisziplinäre Fortbildungen vor Ort angeboten werden.

\section{Kommentar}

Da potenzielle irAEs (immunvermittelte unerwünschte Ereignisse) unter der I-O-Kombination häufig auch subklinisch verlaufen können, sollten nicht nur das Personal an den Zentren, sondern auch die Patienten und ihre Angehörigen dazu angehalten werden, bereits kleinste Veränderungen zu melden. Da das rechtzeitige Nebenwirkungsmanagement die Mitarbeit des Patienten voraussetzt, kommt der Aufklärung des Patienten und seiner Angehörigen bereits im Vorfeld der Therapie eine zentrale Bedeutung zu. Das Behandlungszentrum sollte für den Patienten für eine regelmäßige und gegebenenfalls auch spontane Anreise gut erreichbar sein. Erfahrungsgemäß hat sich zudem die Vorhaltung anderer Angebote wie z.B. ein jederzeit erreichbarer Telefondienst, der direkte E-Mail-Kontakt sowie die Versorgung mit Notfallnummern bewährt (Tab.1).

\section{Disclosure Statement}

Der Autor war Referent beim «Interaktiven Expertenforum Melanom», das von Bristol-Myers Squibb unterstützt wurde. 


\section{Literatur}

1 Schilling B, Weide B: Immuntherapie im Stadium IV in S3-Leitlinie zur Diagnostik, Therapie und Nachsorge des Melanoms, Version 2.0 - Juli 2016; AWMFRegister-Nummer: 032/024OL

2 Larkin J, Chiarion-Sileni V, Gonzalez R, et al: Combined nivolumab and ipilimumab or monotherapy in untreated melanoma. N Engl J Med 2015;373:23-34.

3 Wolchok JD, Chiarion-Sileni V, Gonzalez R, et al: Updated results from a phase III trial of nivolumab combined with ipilimumab in treatment-naïve patients with advanced melanoma (Checkmate 067). J Clin Oncol 2016;34(suppl):abstr 9505.

4 Postow MA, Chesney J, Pavlick AC, et al: Nivolumab and ipilimumab versus ipilimumab in untreated melanoma. N Engl J Med 2015;372:2006-2017.

5 Postow MA, Chesney J, Pavlick AC, et al: Initial report of overall survival rates from a randomized phase II trial evaluating the combination of nivolumab and ipilimumab in patients with advanced melanoma. AACR Annual Meeting 2016; abstr CT002.

6 Ascierto PA, Margolin K: Ipilimumab before BRAF inhibitor treatment may be more beneficial than vice versa for the majority of patients with advanced melanoma. Cancer 2014;120:1617-1619.

7 Ackerman A, Klein O, McDermott DF, et al: Outcomes of patients with metastatic melanoma treated with immunotherapy prior to or after BRAF inhibitors. Cancer 2014;120:1695-1701.

8 Bergelt C, Scholl I, Härter M: Chancen und Barrieren partizipativer Entscheidungsfindung in der Onkologie. Forum 2016;31:140-143.
9 Johnson DB, Sullivan RJ, Ott PA, et al: Ipilimumab therapy in patients with advanced melanoma and preexisting autoimmune disorders. JAMA Oncol 2016;2: 234-240.

10 Weber JS, D'Angelo SP, Minor D, et al: Nivolumab versus chemotherapy in patients with advanced melanoma who progressed after anti-CTLA-4 treatment (CheckMate 037): a randomised, controlled, open-label, phase 3 trial. Lancet Oncol 2015;16:375-384.

11 Ascierto PA, Simeone E, Sileni VC, et al: Sequential treatment with ipilimumab and BRAF inhibitors in patients with metastatic melanoma: data from the Italian cohort of the ipilimumab expanded access program. Cancer Invest 2014;32:144-149.

12 Hugo W, Zaretsky JM, Sun L, et al: Genomic and transcriptomic features of response to anti-PD-1 therapy in metastatic melanoma. Cell 2016;165:35-44.

13 Goldinger SM, Panje C, Nathan P: Treatment of melanoma brain metastases. Curr Opin Oncol 2016;28: 159-165.

14 Goyal S, Silk AW, Tian S, et al: Clinical management of multiple melanoma brain metastases: a systematic review. JAMA Oncol 2015;1:668-676.

15 Sperduto PW, Kased N, Roberge D, et al: Summary report on the graded prognostic assessment: an accurate and facile diagnosis-specific tool to estimate survival for patients with brain metastases. J Clin Oncol 2012;30:419-425.

16 Kortmann RD: Therapie von Hirnmetastasen: Prognostische Faktoren; in S3-Leitlinie zur Diagnostik, Therapie und Nachsorge des Melanoms, Version 2.0 Juli 2016; AWMF-Register-Nummer: 032/024OL.
17 Wick W: Medikamentöse Therapie bei Hirnmetastasen; in S3-Leitlinie zur Diagnostik, Therapie und Nachsorge des Melanoms, Version 2.0 - Juli 2016; AWMF-Register-Nummer: 032/024OL.

18 Koelbl O: Radiotherapie in Kombination mit Ipilimumab; in S3-Leitlinie zur Diagnostik, Therapie und Nachsorge des Melanoms, Version 2.0 - Juli 2016; AWMF-Register-Nummer: 032/024OL.

19 Wilson EH, Weninger W, Hunter CA: Trafficking of immune cells in the central nervous system. J Clin Invest 2010;120:1368-1379.

20 Barker CA, Postow MA: Combinations of radiation therapy and immunotherapy for melanoma: a review of clinical outcomes. Int J Radiat Oncol Biol Phys 2014;88:986-997.

21 Liebner DA, Walston SA, Cavaliere R, et al: Radiation necrosis mimicking rapid intracranial progression of melanoma metastasis in two patients treated with vemurafenib. Melanoma Res 2014;24:172-176.

22 Du Four S, Hong A, Chan M, et al: Symptomatic histologically proven necrosis of brain following stereotactic radiation and ipilimumab in six lesions in four melanoma patients. Case Rep Oncol Med 2014;2014: 417913.

23 Chao ST, Ahluwalia MS, Barnett GH, et al: Challenges with the diagnosis and treatment of cerebral radiation necrosis. Int J Radiat Oncol Biol Phys 2013;87:449457.

24 Aktuelle Fachinformation Opdivo ${ }^{\circledR}$ (Nivolumab).

25 Aktuelle Fachinformation Yervoy ${ }^{\circledR}$ (Ipilimumab). 